\title{
VALORES DE FREQUÊNCIA CARDÍACA DE JOGADORES DE FUTSAL EM SITUAÇÃO DE JOGO
}

\author{
Hélcio Rossi Gonçalves \\ Universidade Paranaense, Umuarama, Paraná, Brasil \\ Wilton Carlos Santana \\ Universidade Estadual de Londrina, Londrina, Paraná, Brasil
}

\begin{abstract}
Resumo
$\mathrm{O}$ artigo tem o objetivo de descrever aspectos relacionados ao valor de frequência cardíaca (FC) de atletas de futsal em situação de jogo, comparando-o com a posição tática. Avaliou-se 16 atletas (24,65 4,25 anos) participantes da Liga Futsal. Os registros de FC foram obtidos por meio de monitores com telemetria. O valor de FC média foi de $123,45 \mathrm{bpm}( \pm 8,33)$ durante o tempo de monitoramento. Em situação de jogo, o valor médio de FC correspondeu à intensidade de $80 \%$ ou $181 \mathrm{bpm}$ $( \pm 7,57)$ sendo o tempo estimado nesta intensidade de $46 \mathrm{~min}( \pm 3,25)$. Quanto às comparações, houve diferenças significativas entre a linha e os goleiros, mas não entre a linha. Conclui-se que a intensidade associada à $\mathrm{FC}$ dos atletas de linha é elevada durante o jogo e deveria ser respeitada no treinamento.
\end{abstract}

Palavras-chave: Futsal. Frequência Cardíaca. Intensidade.

\section{Introdução}

$\mathrm{A}_{\text {tualmente não existem dúvidas da necessidade de se analisarem }}$ tância do princípio da especificidade na elaboração de programas de treinamento, o que resulta da duração, distância e repetição, da carga e velocidade, além de sua frequência de realização, isto é, um aprimoramento das funções para as quais se está treinando (SOARES; TOURINHO FILHO, 2006). Desta forma, a avaliação da demanda metabólica em modalidades esportivas intermitentes, como o futsal, torna-se necessária para a compreensão de como o desempenho e o treinamento devem ser realizados.

Neste sentido, estudar aspectos fisiológicos ligados aos exercícios intermitentes é vital para o entendimento da preparação nos esportes. Reconhecendo ainda que as variações de intensidades e duração dos 
estudos laboratoriais muitas vezes são constantes, o que nem sempre ocorre na prática diária ou competitiva.

Daí acharmos que a característica mais pontual do jogo de futsal seja o dinamismo, traduzido pela marcação intensa, passe acelerado, constante perda e recuperação da posse de bola, movimentações constantes sem a posse da bola, situações de superioridade/inferioridade e igualdade numéricas, diversidade de situações (SANTANA, 2008), solicitações metabólicas intensas, visto que são realizados freqüentes deslocamentos rápidos e curtos, com mudanças de direção e paradas bruscas (ARAÚJO et al, 1996).

Cientes de que planejar um programa de treinamento para um time de futsal é uma tarefa extremamente desafiadora, concordamos com Pinto e Garganta (1996) de que o conhecimento dos modelos de jogo, de preparação e de jogador se torna uma medida acertada, uma vez que estes influenciam, sobremaneira, o planejamento do treino dos JEC.

Neste estudo em particular nos ativemos à dimensão física-condicional, ainda que saibamos que sua manifestação no jogo é dependente das exigências técnico-táticas circunstanciais deste. Logo, somos partidários da ideia de que a dimensão tático-cognitiva, em virtude do complexo sistema de relações existente (jogador-colega-bola-espaçotempo-regras), é nuclear para o desempenho esportivo nos JEC (GARGANTA, 1998), arrastando consigo a necessidade de se imprimir nas diferentes ações mais ou menos força, velocidade etc. (GIMENO, 2007).

Portanto, entendemos que o treinamento das capacidades condicionais será tanto melhor quanto mais próximo da realidade de jogo se encontrar. Considerando, ainda, a máxima do treinar jogando, a ação do treinamento técnico-tático desenvolve, além da capacidade aeróbia dos atletas, os níveis de força durante o macrociclo, já que o futsal apresenta em suas demandas inúmeras ações envolvendo corridas rápidas e de sprint (CETOLIN; FOZA, 2010).

$\mathrm{O}$ fato é que além do aprimoramento técnico-tático (TEJADA; PEÑAS, 2003), a dimensão condicional, isto é, o estado de condicionamento dos jogadores, tem de ser aumentado e mantido em altos níveis. Portanto existe uma ênfase, em todos os níveis do jogo, em desempenhar o máximo possível do treinamento do condicionamento físico nos treinos, isto é, das capacidades motoras particulares do fut- 
sal. Por isso, é necessário compreender as demandas metabólicas da modalidade.

Durante um jogo podemos esperar que as demandas aeróbias alcancem uma média de $70-80 \%$ do consumo máximo de oxigênio. As demandas energéticas durante os períodos de exercício de baixa intensidade, por exemplo, caminhando ou trotando em ritmo leve e durante o período de recuperação de um exercício de alta intensidade, são cobertas pela produção de energia aeróbia. Estas podem ser diretamente determinadas pela medição do consumo de oxigênio, por exemplo, a taxa de consumo de oxigênio pelo corpo. Contudo, o procedimento convencional de coletar o ar exaurido, necessário a esse tipo de medição, evidentemente não é factível para um jogador. A necessidade de energia aeróbia em um jogo de futsal pode ser estimada medindo-se a frequência cardíaca continuamente durante o jogo, baseada na relação direta que existe entre o consumo de energia aeróbia e a frequência cardíaca (GARRETT; KIRKENDALL, 2003).

A maioria da demanda energética durante os períodos de exercício em alta intensidade (por exemplo, sprint, correr com velocidade, pular, chutar) é coberta por produção de energia anaeróbia.

No entanto, é de conhecimento geral que o futsal, assim como outros JEC, caracteriza-se por períodos de alta intensidade e curta duração, intercalados com períodos de baixa intensidade e longa duração, com pausas incompletas, sendo que estes ciclos são extremamente imprevisíveis, resultantes da imposição das diferentes situações que são encontradas durante uma partida e da resposta dos jogadores a essas demandas.

Baseados na importância de estudos da determinação e análise das demandas exigidas durante a realização de uma atividade competitiva, o presente trabalho teve como objetivo analisar e descrever os aspectos relacionados aos valores de frequência cardíaca (FC) obtidos em situação de jogo em jogadores de futsal, comparando-os com a posição/função dos mesmos.

\section{Materiais e métodos}

Para o desenvolvimento deste estudo utilizou-se de uma amostra intencional com 16 jogadores, separados por posição de atuação: seis alas, três pivôs, quatro beques e três goleiros, participantes da Liga Futsal, com média de idade de 24,65 anos $( \pm 4,25)$. Realizou-se a mo- 
nitorizarão da frequência cardíaca mediante um sistema de telemetria portátil, fabricado pela Polar Eletronic, modelo "Polar - Acurex Plus" em três jogos amistosos. Esse sistema consiste de um pequeno transmissor com dois eletrodos, fixado na parte anterior do tronco, e um receptor utilizado como relógio de pulso, que foi fixado junto ao transmissor, para evitar qualquer tipo de lesão ao atleta ou mesmo ao adversário, capaz de armazenar os sinais emitidos pelos batimentos cardíacos para futura recuperação através de interface computadorizada (LEGER; THIVIERGE, 1988).

Os atletas utilizaram os monitores durante todo o tempo da atividade, incluindo: aquecimento, os jogos propriamente ditos, intervalos e recuperações, substituições e tempos. O tempo médio de utilização do equipamento foi de $115,38 \mathrm{~min}( \pm 11,21 \mathrm{~min})$. Durante este período foram registradas as atividades que os atletas realizaram, através de uma ficha construída especificamente para esse fim. Outro procedimento adotado foi verificar o tempo em que os atletas permaneciam em intensidades predeterminadas de exercícios, ou seja, FC Min $(<$ 40\%), FC Média (40\% - 80\%) e FC Max (> 80\%) conforme estabelecem os parâmetros de treinamentos (DENADAI; GRECO, 2005).

Análise estatística

Quanto aos procedimentos estatísticos, utilizaram-se informações da estatística descritiva, além do teste de comparações múltiplas "POST-HOC" de Scheffé, que foi empregado na identificação das diferenças específicas quando das comparações dos jogadores por posição de atuação no jogo com os valores de significância estatísticos estabelecidos ao nível de $\mathrm{p}<0,01$ e $\mathrm{p}>0,01 \mathrm{p}<0,05$. (THOMAS; NELSON, 2002)

\section{Resultados}

A análise dos resultados foi dividida em dois momentos: inicialmente, levando-se em consideração as médias dos valores encontrados de FC com as divisões em FC Média, FC Mínima e FC Máxima alcançadas durante os esforços; posteriormente, relacionando-as com os tempos em minutos de permanência em quadra dos jogadores em cada uma das intensidades. Outro aspecto a ser considerado nesses dois itens se refere à posição de atuação/função dos jogadores. 
Quando os dados foram analisados de forma geral, levando-se em consideração os valores de FC de todos os atletas, percebemos que os valores médios de FC Média durante todo o tempo da atividade (Tabela 1) foi em média de 123,45 bpm ( $\pm 8,33 \mathrm{bpm})$, o que deve ser relacionado com o tempo médio de permanência nessa frequência, ou seja, de aproximadamente $45,68 \mathrm{~min} .( \pm 3,42)$ de todo o tempo de monitoramento dos atletas. Já as informações de FC Máxima, tiveram valores de $181,00 \mathrm{bpm}( \pm 7,57)$ e o tempo médio de permanência nessa frequência foi de $45,64 \min ( \pm 3,25)$.

Tabela 1: Valores de média e desvio padrão das informações de FC e dos tempos em minutos em que os jogadores permaneceram monitorados nas respectivas intensidades por posição de atuação e da equipe.

\begin{tabular}{|c|c|c|c|c|c|c|}
\hline & & $\begin{array}{l}\text { Pivôs } \\
\mathrm{n}=3\end{array}$ & $\begin{array}{c}\text { Beques } \\
\mathrm{n}=4\end{array}$ & $\begin{array}{l}\text { Alas } \\
\mathrm{n}=6\end{array}$ & $\begin{array}{c}\text { Goleiros } \\
\mathrm{n}=3\end{array}$ & $\begin{array}{c}\text { Equipe } \\
\mathrm{n}=16\end{array}$ \\
\hline \multirow{4}{*}{$\begin{array}{l}\text { FC Min } \\
(<40 \%)\end{array}$} & Valores de & 69,67 & 69,50 & 75,43 & 75,00 & 72,40 \\
\hline & FC & $\pm 3,79$ & $\pm 3,21$ & $\pm 7,61$ & $\pm 4,93$ & $\pm 4,88$ \\
\hline & Tempos na & 23,15 & 21,26 & 22,38 & $29,44^{\mathrm{b}}$ & 24,05 \\
\hline & FC & $\pm 2,49$ & $\pm 3,25$ & $\pm 3,58$ & $\pm 2,93$ & $\pm 3,17$ \\
\hline \multirow{4}{*}{$\begin{array}{c}\text { FC Média } \\
(41 \%-80 \%)\end{array}$} & Valores de & 130,46 & 126,79 & 126,56 & $110,00^{b}$ & 123,45 \\
\hline & FC & $\pm 6,40$ & $\pm 7,15$ & $\pm 10,97$ & $\pm 8,80$ & $\pm 8,33$ \\
\hline & Tempos na & 42,18 & 43,65 & $35,43^{b}$ & $61,49^{a}$ & 45,68 \\
\hline & FC & $\pm 2,36$ & $\pm 3,18$ & $\pm 3,51$ & $\pm 4,25$ & $\pm 3,42$ \\
\hline \multirow{5}{*}{$\begin{array}{l}\text { FC Max } \\
(>81 \%)\end{array}$} & Valores de & 185,00 & 189,00 & 185,00 & $165,00^{\mathrm{a}}$ & 181,00 \\
\hline & FC & $\pm 2,65$ & $\pm 5,73$ & $\pm 9,87$ & $\pm 12,04$ & $\pm 7,57$ \\
\hline & Tempos na & 56,56 & $48,29^{b}$ & 52,14 & $25,57^{a}$ & 45,64 \\
\hline & FC & $\pm 3,14$ & $\pm 2,65$ & $\pm 4,17$ & $\pm 3,12$ & $\pm 3,25$ \\
\hline & $\begin{array}{c}\text { Tempo total } \\
\text { (min) }\end{array}$ & 121,89 & 113,20 & 109,95 & 116,50 & 115,38 \\
\hline
\end{tabular}

$\mathrm{a}=\mathrm{p}<0,01 \overline{\mathrm{b}=\mathrm{p}>0,01 \mathrm{p}<0,05 \text { - diferenças significativas quanto às compara- }}$ ções nas posições de atuação.

Na Tabela 2, é possível verificar a porcentagem de tempo em que os atletas permaneceram nas diferentes intensidades, ficando mais uma vez evidente as diferenças das demais posições em relação aos goleiros. Igualmente, se pode observar que em aproximadamente $40 \%$ do tempo de monitoramento os esforços físicos são executados a intensidades máximas. 
Tabela 2: Valores percentuais de tempo em que os jogadores permaneceram monitorados nas respectivas intensidades por posição de atuação e da equipe.

\begin{tabular}{c|c|c|c|c|c}
\hline Posição (n)/ & Pivôs & Beques & Alas & Goleiros & Equipe \\
\cline { 2 - 6 } Intensidades & $\mathrm{n}=3$ & $\mathrm{n}=4$ & $\mathrm{n}=6$ & $\mathrm{n}=3$ & $\mathrm{n}=16$ \\
\hline FC Min (<40\%) & 18,99 & 18,78 & 20,35 & 25,27 & 20,84 \\
\hline FC Média (41\%- 80\%) & 34,60 & 38,56 & 32,22 & 52,78 & 39,59 \\
\hline FC Max (> 81\%) & 46,40 & 42,66 & 47,42 & 21,95 & 39,57 \\
\hline
\end{tabular}

Nas figuras 3 é possível verificar as informações de FC Mínima, Média e Máxima de cada um dos jogadores pesquisados.

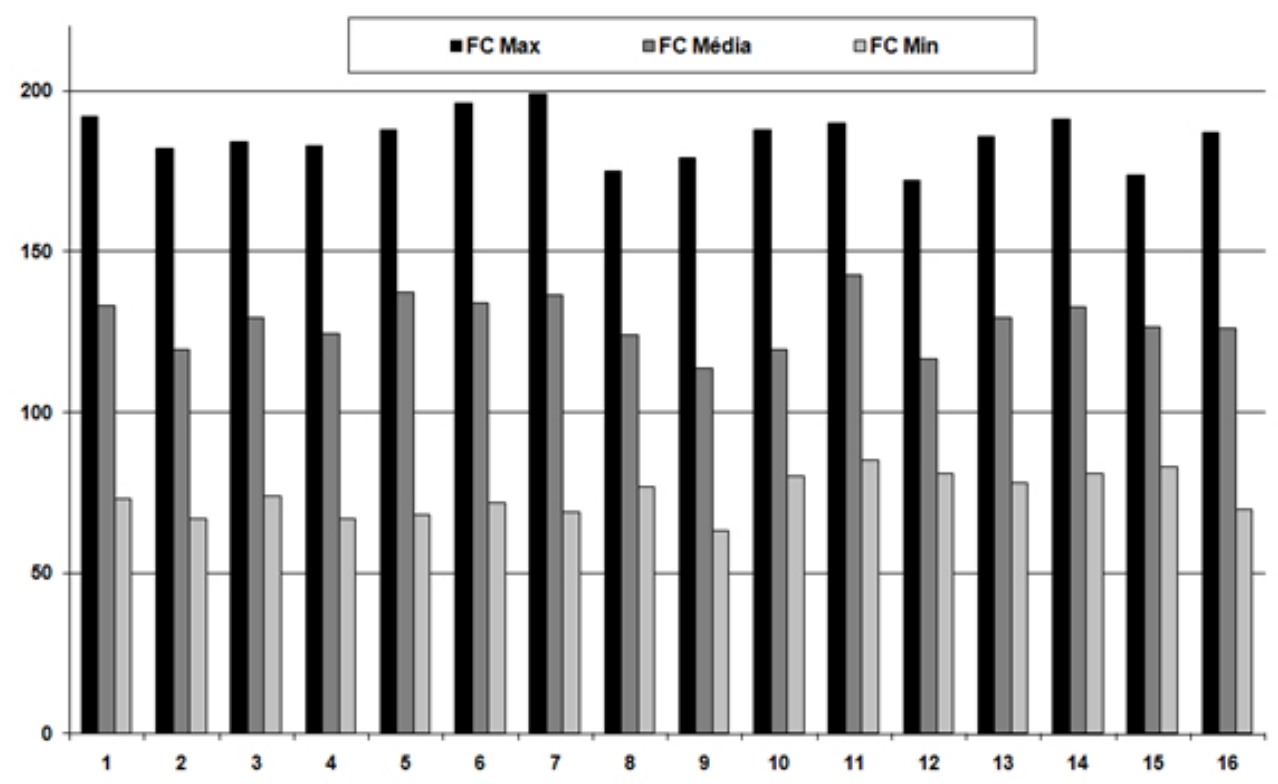

Figura 3: Com valores individuais dos jogadores com informações de FC Média, FC Máxima e Mínima. 


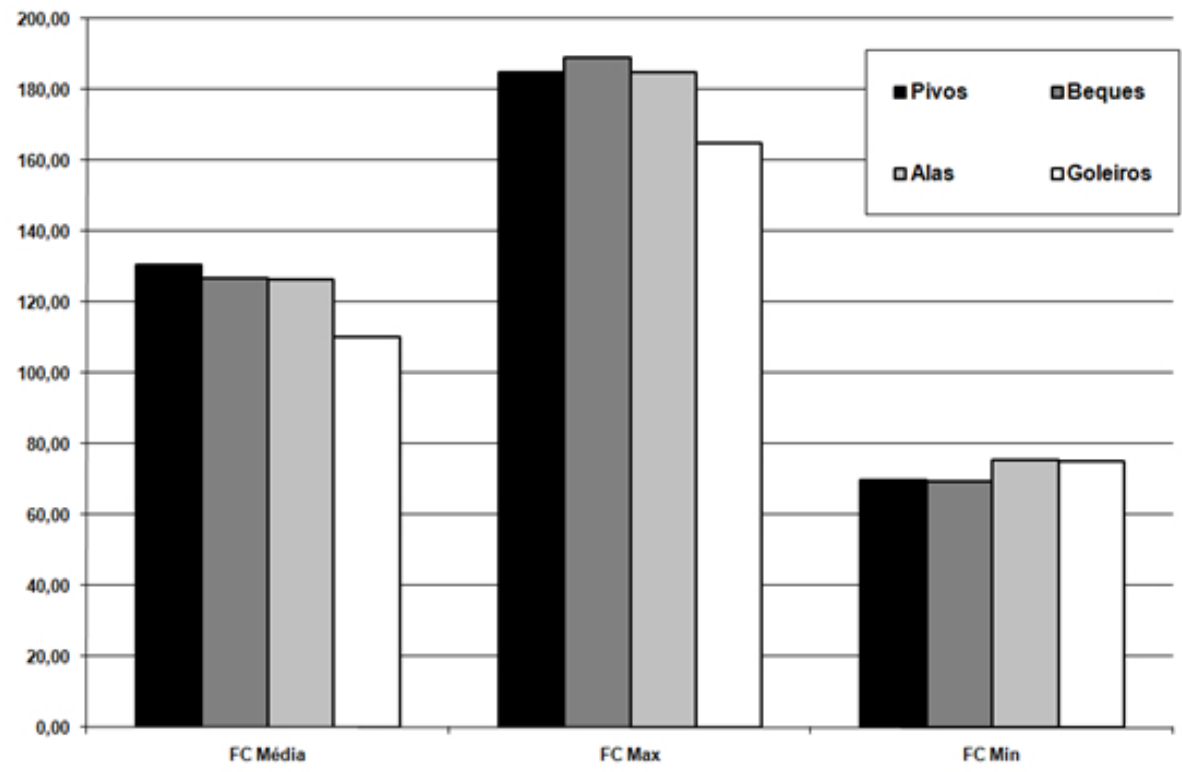

Figura 4: Valores das médias das FC médias, FC mínimas e máximas, por posição e atuação em jogo.

A figura 4 contempla os valores das médias das FC médias, FC mínimas e máximas separadamente por posições de atuação em jogo, sendo possível perceber que as diferenças mais evidentes quanto as diferentes posições esta associada aos demais jogadores e os goleiros.

\section{Discussão}

Considerando a iniciativa deste trabalho de analisar e descrever os aspectos relacionados aos valores de frequência cardíaca (FC) obtidos em situação de jogo em jogadores de futsal, e comparação dos jogadores quanto a suas posições e funções no jogo, tornou-se possível medir a carga de trabalho imposta durante as partidas.

A determinação de intensidades, vias metabólicas predominantes, assim como outras características ligadas ao treinamento, são relevantes para que possam ocorrer ajustes/adaptações fisiológicas, devendo haver limites de intensidade mínimos, abaixo dos quais não se processará nenhum efeito de treinamento, assim como tetos máximos, em que seria desnecessário se exercitar acima destes para se obter mais eficácia em determinadas vias energéticas (DENADAI; GRECO, 2005). Logo, sugere-se a utilização de um limiar de treinabilidade (LT) para estimar a frequência cardíaca de treinamento (FCT), o que 
também pode ser associado a valores de Limiar Anaeróbio (GUERRA; BARROS, 2004; FERREIRA, et al., 2008), que seria fundamental ao processo de treinamento de uma equipe e, por sua vez, de cada jogador.

Atualmente não existem dúvidas quanto a necessidade de se analisarem as demandas metabólicas de atividades competitivas, principalmente aquelas de caráter intermitentes, como é caso do futsal, o que possibilitaria melhorar a compreensão sobre como o treinamento deve ser realizado. O padrão de movimento de um jogador de futsal durante o jogo é influenciado por numerosas variáveis, tais como o nível da competição, exigências táticas impostas ao jogador e à equipe, tática do time adversário, resultado do jogo, condições ambientais etc. Todavia, como mencionado anteriormente, o futsal pode ser caracterizado como uma modalidade de solicitação intermitente, com predominância anaeróbica lática. Fato que pode ser comprovado por informações de concentração de lactato em atletas (MONTEIRO, et al., 2009), ou ainda caracteriza-se por ser atividade de esforços intermitentes que mesclam na sua execução a participação tanto do metabolismo aeróbio como do anaeróbio, o que também pode ser comprovado pela variabalidade de FC apresentada neste estudo.

Considerando essas informações, alguns estudos têm procurado determinar valores de Frequência Cardíaca que possam auxiliar no processo de treinamento. Medina et al. (2002) obtiveram valores médios de 165 bpm e máximos e mínimos de 181-141bpm, e intensidade de aproximadamente 85 a $90 \%$ da FC máxima foram encontrados para jogadores dessa modalidade, enquanto Álvarez et al. (2004) obtiveram valores ligeiramente superiores, sendo a média de 172,9 bpm e 192 bpm e $118 \mathrm{bpm}$, para máximo e mínimo respectivamente. Maclaren (1988) monitorou a FC durante o jogo (172 bpm), representando um VO2max de 3.951. min- ${ }^{1}$, equivalente a uma intensidade de trabalho de $82 \%$ VO2max, estimando um gasto energético em 79,4 kJ. Min.- ${ }^{-}$, enquanto que Molinuevo e Ortega (1989) apresentaram FC de 138 bpm com atletas espanhóis.

De forma geral, os resultados são similares quanto aos valores máximos aqui obtidos, contudo os modelos metodológicos dos estudos apresentam algumas diferenças. Neste sentido, demonstraram desigualdades quanto aos valores mínimos e, por conseqüência, os escores médios, uma vez que a análise deste estudo considerou, também, va- 
lores iniciais de aquecimento e recuperações durante e no final dos jogos.

Quando separamos os jogadores por posição de atuação (Tabela 1), ou seja, alas, pivôs, beques e goleiros, percebeu-se que não houve diferenças significativas entre os jogadores de linha (alas, beques, pivôs). Porém, essa diferença existiu quando comparados com os goleiros. De certa forma isso ocorreu praticamente em todas as análises com os goleiros, seja de Valores de FC ou de Tempo nas FCs, exceto nos valores de FC Mínima, o que acreditamos ser plausível em função das exigências da posição. Enquanto que com os jogadores de linha tais diferenças só ficaram evidentes com os alas e beques nas informações de FC Média e FC Máxima, respectivamente.

Evidências similares também foram obtidas por Soares e Tourinho Filho (2006), ao demonstrarem que as atividades de baixa intensidade predominaram na posição de goleiro; as atividades de média intensidade, na posição do ala; as de alta intensidade, com os beques.

Quanto ao tempo total de monitoramento dos atletas, a variação é pequena e acreditamos que isso tenha contribuído para uma analise mais crítica quanto às diferentes posições de atuação em jogo, favorecendo, dessa forma, as comparações entre as posições.

Ressaltamos que alguns atletas permaneceram em quadra mais tempo do que normalmente ocorreria em uma partida oficial, fato também observado em outro estudo (ARAUJO, et. al., 1996). Isso, em alguns casos, pode ter superestimado os valores de FC Máxima de jogadores em suas posições de atuação. Contudo, acreditamos que a maior contribuição do estudo tenha sido a possibilidade de se estabelecerem intensidades de treinamento, uma vez que é amplamente citado na literatura a relação existente entre os valores de $\mathrm{VO} 2$ max. e FC (ACMS, 2000; FRANKLIN; HODGSON; BUSKIRK, 1980; McARDLE; KATCH; KATCH, 2003; TANAKA, MONAHAN; SEALS, 2001).

Levando-se em consideração que a idade média dos jogadores deste estudo é de 24,65 anos e considerando a equação 220-idade, os valores médios de FC Máxima seriam de aproximadamente 195,35 bpm (ROBERGS, R.A.; LANDWEHR, 2002), e desta forma, estariam treinando a uma intensidade aproximada de 90 a $95 \%$ de sua capacidade máxima. E, finalmente, as informações de FC Mínima, de 72,40 $\operatorname{bpm}( \pm 4,88)$ e $24,05 \min ( \pm 3,17)$. Contudo, esses valores são agregados principalmente às informações de recuperação dos jogadores, $\mathrm{o}$ 
que acreditamos, por algumas estimativas em situações vivenciadas em análises de scout do campeonato, seja de aproximadamente 3 minutos.

\section{Conclusão}

No que se refere aos valores de $\mathrm{FC}$ da equipe e dos jogadores por posição de atuação no jogo, pôde-se perceber que os atletas de linha apresentam valores diferenciados dos goleiros. No entanto, entre eles (alas, pivôs e beques), essas diferenças não existem de forma significativa. Acrescente-se que as intensidades de demanda energéticas associadas à FC são se demonstraram, neste estudo, elevadas durante uma partida.

Quanto aos valores associados ao tempo em que os jogadores se mantiveram nas FC Mínima, Média e Máxima, nos parece pertinente que os atletas de linha devem ter características de treinamento diferenciadas dos goleiros, de forma que fiquem condizentes com os valores encontrados. Desta forma acreditamos que isso seria primordial para a obtenção de ajustes satisfatórios quanto ao condicionamento desses atletas, bem como para sua recuperação durante o jogo.

Imaginamos que o estudo seja útil a preparadores físicos e treinadores, para que possam usá-lo como referência a fim de estabelecerem intensidades de treinamento que sejam apropriadas ao dinamismo do jogo de equipes profissionais, embora não tenham sido levados em consideração fatores estratégico-táticos, como o tipo de marcação, os princípios ofensivos etc., que podem alterar as demandas físico-condicionais.

Por último, acreditamos que o desenvolvimento de novos estudos sobre o assunto, bastante escasso em nossa literatura, em particular com um número maior de jogadores e equipes de diferentes níveis, contribuiria para um maior entendimento do tema.

\section{Value of heart rate of in door soccer in game situation}

\section{Abstract}

This article objective to describe aspects related to the value of heart rate (HR) of indoor soccer players in game situation, comparing it with the tactical position. We evaluated 16 athletes $(24.65 \pm 4.25$ years) participating in the Indoor Soccer League. HR records were obtained from monitors with telemetry. The average HR 
value was $123.45 \mathrm{bpm}( \pm 8.33)$ during the monitoring time. In game situation, the average HR corresponded to the intensity of $80 \%$ or $181 \mathrm{bpm}( \pm 7.57)$ and the estimated time of $46 \mathrm{~min}$ at this intensity $( \pm 3.25)$. As for comparisons, significant differences between the line and the goalkeepers, but not across the line. It is concluded that the intensity of the line athletes associated with HR is high during the game and should be respected in the training.

Keywords: Indoor Soccer. Heart Rate. Intensity.

Valores de frecuencia cardiaca de los jugadores de fútbol sala en el juego

\section{Resumen}

El artículo pretende describir aspectos del valor de la frecuencia cardíaca (FC) de atletas de futsal en situación de juego, comparándolo con la posición táctica. $\mathrm{Si}$ evaluaron 16 atletas ( $24.65 \pm 4,25$ años) participando en la Liga Futsal. Registros FC se obtuvieron a través de monitores con telemetría. El valor de FC promedio fue de $123,45 \mathrm{bpm}( \pm 8,33)$ durante el rastreo de tiempo. En el juego, el valor promedio de FC correspondía a la intensidad de $80 \%$ o $181 \mathrm{bpm}( \pm 7.57)$ que estima esta intensidad tiempo de 46 minutos $( \pm 3,25)$. Con respecto a las comparaciones, hubo diferencias significativas entre la línea y los porteros, pero no a través de la línea. Se llegó a la conclusión de que la intensidad asociada a FC de los atletas de la línea es alta durante el juego y deben respetarse en la práctica.

Palabras clave: Futsal. Frecuencia Cardíaca. Intensidad.

\section{Referências}

ÁlVAREZ, J. C. B.; VERA, J. G.; HERMOSO, V. M. S. Análisis de La frecuencia cardíaca durante La competición em jugadores profesionales de fútbol sala. Apunts: Educación física y deportes, n. 77 p. 71$78,2004$.

AMERICAN COLLEGE OF SPORTS MEDICINE - ACMS. Guidelines for exercise testing and prescription. 6. ed. Philadelphia: Lippincott Williams \& Wilkins, 2000.

ARAUJO, T. L. et al. Demanda fisiológica durante o jogo de futebol de salão, através de distancia percorrida. Revista da Associação dos Professores de Educação Física de Londrina, Londrina, v. 11, n. 19, p. 12-20, 1996.

BALSOM, P. D. Futebol de Precisão. Finlândia: Polar Electro OV, 2001. 
CETOLIN, T.; FOZA, V. Periodização no futsal: descrição da utilização da metodologia de treinamento baseada nas cargas seletivas. Brazilian Journal of Biomotricity, v. 4, n.1, p.24-31, mar., 2010.

DANTAS, E. H. M. A Pratica da Preparação Física. 2. ed. Rio de Janeiro: Shape, 1998.

DENADAI, B. S.; GRECO, C. C. Prescrição do Treinamento Aeróbio: teoria e prática. Rio de Janeiro: Guanabara Koogan, 2005.

FERREIRA, A. P. et al. Composição corporal, limiar anaeróbio e consumo máximo de oxigênio de atletas de Futsal: analise descritiva entre as posições. Revista Brasileira de Ciência e Movimento, Brasília, v.16, n. 3, p.60 - 68, 2008.

FRANKLIN, B.A.; HODGSON J.; BUSKIRK, E.R. Relationship between percent maximal $\mathrm{O} 2$ uptake and percent maximal heart rate in women. Research Quarterly for Exercise and Sport, v. 51, n. 4, p. 616-624, 1980.

GARGANTA J. Para uma teoria dos jogos desportivos colectivos. In: GRAÇA, A.; OLIVEIRA, J. O ensino dos jogos desportivos. 3. ed. Porto: FCDEFUP, 1998. p.11-25.

GARRETT, W. E.; KIRKENDALL, D. T. Ciência do Exercício e dos Esportes. Porto Alegre: Artmed, 2003.

GIMENTO, T. X. Futbol que es la periodizacion táctica?. Madri: Moreno y Conde Sports, S.L. 2007.

GOMES, A. C. Treinamento Desportivo: Estruturação e Periodização. Porto Alegre: Artmed, 2002.

GOMES, A C, MACHADO, J. A. Futsal: metodologia e planejamento na infância e adolescência. Londrina: Midiograf, 2001.

GUERRA, I.; BARROS, T. L. Ciência do Futebol. Barueri: Manole, 2004.

KARVONEN, M. J.; KENTALA, E.; MUSTALA, O. The effects of training on heart rate: a longitudinal study. Annales Medicinae Experimentalis et Biologiae Fenniae, v. 35, n. 3, p. 307-315, 1957. 
LEGER, L.; THIVIERGE, M. Heart rate monitors: validity, stability and functionality. Physician and Sports Medicine, v.16, p.143-51, 1988.

MACLAREN, D. Physiological strain in 4-a-side soccer. In: REILLY, $\mathrm{T}$ et al. Science and Football. London : E; F.N. Spon, 1988. p. 76-80.

McARDLE, D. W.; KATCH, I. F.; KATCH, L. V. Fisiologia do Exercício Energia, Nutrição e Desempenho Humano. 5. ed. Rio de Janeiro: Guanabara Koogan, 2003.

MEDINA, J. A. et al. Necessidades cardiovasculares y metabólicas del fútbol sala: análisis de La competición. Apunts: Educación física y deportes. n. 67, p. 45-53. 2002.

MOLINUEVO, J. S.; ORTEGA, A. M. Perfil morfofuncional de uma equipe de futebol de salão. In: CONGRESSO INTERNACIONAL SOBRE CIÊNCIA E TÉCNICA DE FUTEBOL, Madri, 1989, Anais... Madri: 1989. p.217-224

MONTEIRO, A. G. et al. Respostas cardiorrespiratórias e metabólicas em partidas do campeonato paulista de futsal na categoria adulto. 2009. Disponível em <http://www.arturmonteiro.com.br/wpcontent/uploads/2009/02/artigo-respostas-fisiologicas-no-futsal.pdf $>$. Acesso em 08 nov. 2011.

MORENO, J. H. Fundamentos del deporte: análisis de las estructuras del juego deportivo. 2. ed. Barcelona: INDE, 1998.

PINTO, J.; GARGANTA, J. Futebol: importância do modelo de jogo no seu desenvolvimento. Revista Horizonte. n.33, p.94-98, 1996.

ROBERGS, R. A.; LANDWEHR, R. The surprising history of the "HRmax=220-age" equation. Journal of Exercise Physiology online, v. 5, n. 2, p. 18-25, 2002.

SANTANA, W. C. O raciocínio do treino. In: Futsal: apontamentos pedagógicos na iniciação e na especialização. 2. ed. Campinas: Autores Associados, 2008. p. 129-132.

SOARES, B.H.; TOURINHO FILHO, H. Análise da distância e intensidade dos deslocamentos, numa partida de futsal, nas diferentes 
posições de jogo. Revista Brasileira de Educação Física e Esporte, São Paulo, v.20, n.2, p.93-101, abr./jun. 2006.

TANAKA, H.; MONAHAN, K. D.; SEALS, D. R. Age: predicted maximal heart rate revisited. Journal of the American College of Cardiology, v. 37, n. 1, p. 153-156, 2001.

TEJADA, J. V, PEÑAS, J. L. Entrenamiento de base en fútbol sala: fundamentos teóricos e aplicaciones prácticas. Barcelona: Paidotribo, 2003.

THOMAS, J. R.; NELSON, J. K. Métodos de pesquisa em atividade física. 3. ed. Porto Alegre: Artmed, 2002.

TUBINO, M. J. G. Metodologia Cientifica do Treinamento Desportivo. 3. ed. São Paulo: Ibrasa, 1994.

WEINECK, J. Treinamento Ideal. 9. ed. São Paulo: Manole, 1999.

Recebido em: 17/11/2011

Revisado em: 27/01/2012

Aprovado em: 17/04/2012

Endereço para correspondência

wilton@pedagogiadofutsal.com.br

Wilton Carlos de Santana

Universidade Estadual de Londrina

Centro de Educação Física e Desportos.

Rodovia Celso Garca Cid, km 380

Campus Universitário

86055-900 - Londrina, PR - Brasil 